\title{
A MODEL OF MULTIPLE MAGNETIC SEPARATION IN THE CONTINUOUS PROCESS
}

\author{
M. BROŻEK \\ University of Mining and Metallurgy, Al. Mickiewicza 30 , \\ 30-059 Cracow, Poland
}

(Received 13 August 1998; Accepted 26 October 1998)

In continuous enrichment processes, in industrial conditions and due to the generally required high efficiency of machines, a relatively high concentration of feed is applied. Since it is necessary to liberate the useful minerals, the feed has to be ground thoroughly and this fact results in the percentage growth of the fraction of particles measuring a few tens or several micrometers. When the particles are so fine and the feed is so much concentrated, the magnetic particles interactions play a significant role and they lead to the phenomenon of magnetic flocculation. The flocs contain the particles of waste rock and intergrowths. In order to obtain the final product of high quality the material is subjected to multiple enrichment operations.

This paper presents a mathematical model of multiple magnetic separation in which the grade of the magnetic concentrate was expressed as the function of multiplication ratio of separation and the value of external forces acting on a particle as well as interactions of the magnetic particles. The dependence of the content of magnetic component in the highgradient separation concentrates on the multiplication ratio of separation, flow velocity of the suspension through the separator working space, the intensity of matrix vibration and the concentration of solid particles in the feed for the separation of magnetite ore was verified. The verification was carried out according to the author's own research and the reference.

Keywords: Magnetic separation; Mathematical model; Multistage separation; Magnetic interactions

\section{INTRODUCTION}

Industrial processes of magnetic separation, due to their mass character and the necessity of enrichment of large quantities of the throughput, are carried out in a continuous way. In the course of the process the 
feed is continuously transported to the separator and the enrichment products are also continuously collected. These are: the magnetic product and the non-magnetic product. Since it is necessary to liberate the useful minerals, the feed for the enrichment process must be first crushed to the range of a few tenths of a millimeter. The material, crushed in such a way, is subjected to the action of magnetic field in the separator working space. Under the influence of the magnetic field the magnetic particles obtain magnetic moments whose mutual interactions lead to the phenomenon of magnetic flocculation. The non-magnetic particles are bound in the internal structure of flocs by magnetic, surface and mechanical forces.

If the separation is performed in the constant magnetic field, the nonmagnetic particles are transported, together with the magnetic component, to the magnetic product, decreasing the quality of this product. Due to that, in order to obtain the final product of high quality, the material should be subjected to multiple separations (cleaning operations). When the material of high magnetic susceptibility, such as, for instance, magnetite ore, is enriched, the magnetic product is sometimes subjected, between enrichment operations, to demagnetization in order to break the floc structure. During each consecutive separation (stage) a certain portion of the non-magnetic component is removed from the enriched material.

In the process of cyclic separation the magnetic component, due to the forces of the magnetic field, is removed from working chamber of the separator while the non-magnetic component remains in the working chamber during the entire process. The amount of the separated magnetic component is the function of time and the concentration of this component in the separator working space changes in time. Transporting the magnetic component outside working space of the separator in the process of the cyclic separation is a pure death process from the point of view of Markovian stochastic processes [1]. The duration of the separation process may be arbitrarily long. Even if magnetic particle returns to the working chamber of the separator from the magnetic product, it can be brought again to the magnetic product in the next time interval. Because of that, the entire amount of the magnetic component can be separated in the cyclic process.

As opposed to the cyclic separation, in the continuous process, the chance of return of the magnetic particle to the magnetic product is 
slight, due to the short residence time the material in the working chamber of the separator. Because of that, magnetic separation in the continuous process is a process of birth and death [1]. In this system only a part of the magnetic component can be separated. With fixed and time independent distributions of magnetic properties of feed particles and with fixed capacity and separation conditions the relative amount of the separated magnetic component does not depend on time.

\section{A MODEL OF MULTIPLE MAGNETIC SEPARATION}

As it was mentioned at the very beginning, the process of multiple separation proceeds in a continuous way. A magnetic product from the separation in the $k$ th stage constitutes a feed for separation in the $(k+1)$ th stage. In every stage the material, in the magnetic field zone, travels a certain distance along the separator working space, called the separation path. In the process of multiple separation the effective length of the separation path will be proportional to the number of stages The effective path is a path travelled by the material in the magnetic field zone.

The subject-matter of the considerations will be constituted by the separation of the non-magnetic component of susceptibility $\chi_{\mathbf{n}}$ from the stream of magnetic product (main stream). In every stage a certain amount of non-magnetic component is carried off.

Let $r$ denote an effective length of the separation path. The value

$$
P(r>s)=f(s)
$$

will denote the probability of the non-magnetic particle remaining in the main material stream to a point $s$ or, in a different way, the probability of non-separation of the non-magnetic particle into the non-magnetic product on the separation path $s$ when the condition

$$
f(0)=P(r>0)=1
$$

is fulfilled.

A non-separation of the non-magnetic particle in two consecutive sections of the effective path $s$ and $u(s, u \geq 0)$ will be a pair of independent events. Therefore the probability of non-separating the 
non-magnetic particle in the joint section of the effective path $s+u$ will be, according to the formula of total probability, equal to [2]

$$
\begin{aligned}
P(r>s+u) & =P(r>u) P(r>s), \\
f(s+u) & =f(u) f(s) .
\end{aligned}
$$

After differentiating expression (3b) in relation to $u$ (assuming that there is a density $\left.f^{\prime}(s+0), s \geq 0\right)$, we can obtain

$$
f^{\prime}(s+u)=f(s) f^{\prime}(u) .
$$

Dividing both sides of Eq. (3b) by Eq. (4) we obtain

$$
\frac{f(s+u)}{f^{\prime}(s+u)}=\frac{f(u)}{f^{\prime}(u)}
$$

in which at $u=0$

$$
\frac{f^{\prime}(s)}{f(s)}=-\mu
$$

where $-\mu=f^{\prime}(0) \leq 0$ because $f(0)=1$ is the maximum value of function $f(s)=P(r>s), s \geq 0$. From Eq. (6) after considering condition (2) we obtain the following solution

$$
f(s)=\mathrm{e}^{-\mu s} .
$$

As it was said before, $f(s)$ denotes a probability of non-separation of the non-magnetic particle of susceptibility $\chi_{\mathbf{n}}$ into the non-magnetic product. The measure of this probability will be constituted by a number of particles of susceptibility $\chi_{\mathrm{n}}$, contained in the magnetic product after travelling the path $s$ (number of non-separated particles) to the total number of particles of this susceptibility in the feed

$$
f\left(s \mid \chi_{\mathrm{n}}\right)=\frac{N_{\mathrm{m}}\left(s \mid \chi_{\mathrm{n}}\right)}{N\left(0 \mid \chi_{\mathrm{n}}\right)}
$$

Therefore from expression (7) after considering Eq. (8) we obtain

$$
N_{\mathrm{m}}\left(s \mid \chi_{\mathrm{n}}\right)=N\left(0 \mid \chi_{\mathrm{n}}\right) \mathrm{e}^{-\mu s}
$$


In the above equations $N_{\mathrm{m}}\left(s \mid \chi_{\mathrm{n}}\right)$ denotes the number of non-magnetic particles of susceptibility $\chi_{\mathrm{n}}$, contained in the magnetic product after travelling the path $s$ while $N\left(0 \mid \chi_{\mathrm{n}}\right)$ denotes the number of particles of susceptibility $\chi_{\mathrm{n}}$ in the feed.

The value

$$
N_{\mathrm{n}}\left(s \mid \chi_{\mathrm{n}}\right)=N\left(0 \mid \chi_{\mathrm{n}}\right)-N_{\mathrm{m}}\left(s \mid \chi_{\mathrm{n}}\right)=N\left(0 \mid \chi_{\mathrm{n}}\right)\left(1-\mathrm{e}^{-\mu s}\right)
$$

represents the number of particles of susceptibility $\chi_{n}$ separated to the non-magnetic product after travelling the path $s$ by the main stream. The total recovery of these particles in the non-magnetic product (to the point $s$ of the effective path of the main stream) will be equal to

$$
\varepsilon\left(s \mid \chi_{\mathrm{n}}\right)=\frac{N_{\mathrm{n}}\left(s \mid \chi_{\mathrm{n}}\right)}{N\left(0 \mid \chi_{\mathrm{n}}\right)}=1-\mathrm{e}^{-\mu s}
$$

If the length of the separation path in a single stage is equal $s_{1}$, then after $k$ stages the effective separation path will be $s=s_{1} k$. In such a situation expression (11) can be written as follows:

$$
\varepsilon\left(k \mid \chi_{\mathrm{n}}\right)=1-\exp \left(-\mu s_{1} k\right)
$$

The length of the separation path in one stage $s_{1}$ depends on the type and dimensions of the working space of the separators applied in a given technological process.

It results from formula (12) that with the growing number of stages (cleaning separations) the recovery of the non-magnetic component into the non-magnetic product is growing and consequently the residue of this component in the magnetic product is decreasing.

\section{TECHNOLOGICAL INDICES OF SEPARATION}

The residue of the non-magnetic component in the magnetic product after $k$ separations is equal to

$$
\sigma\left(k \mid \chi_{\mathrm{n}}\right)=1-\varepsilon\left(k \mid \chi_{\mathrm{n}}\right)=\exp \left(-\mu s_{1} k\right) .
$$


On the other hand, the residue of this component in the magnetic product is expressed [3] by

$$
\sigma\left(k \mid \chi_{\mathrm{n}}\right)=\frac{\gamma_{\mathrm{m}}(k) \lambda_{\mathrm{n}}\left(k \mid \chi_{\mathrm{n}}\right)}{\alpha_{\mathrm{n}}\left(\chi_{\mathrm{n}}\right)}
$$

where $\gamma_{\mathrm{m}}(k)$ is the yield of the magnetic product after $k$ stages of separation, $\lambda_{\mathrm{n}}\left(k \mid \chi_{\mathrm{n}}\right)$ the content of the non-magnetic component in the magnetic product, and $\alpha_{\mathrm{n}}$ the content of the non-magnetic component in the feed.

The balance equation for the non-magnetic component at the division of the feed into two products, i.e. magnetic and non-magnetic, is as follows:

$$
\alpha_{\mathrm{n}}=\gamma_{\mathrm{m}} \lambda_{\mathrm{n}}+\left(1-\gamma_{\mathrm{m}}\right) \vartheta_{\mathrm{n}}
$$

where $\vartheta_{\mathrm{n}}$ is the content of the considered non-magnetic component in the non-magnetic product. From Eqs. (13)-(15) the concentration of the non-magnetic component in the magnetic product is

$$
\lambda_{\mathrm{n}}\left(k \mid \chi_{\mathrm{n}}\right)=\frac{\vartheta_{\mathrm{n}} \alpha_{\mathrm{n}} \exp \left(-\mu s_{1} k\right)}{\vartheta_{\mathrm{n}}-\alpha_{\mathrm{n}}+\alpha_{\mathrm{n}} \exp \left(-\mu s_{1} k\right)} .
$$

For the two-component mixture, composed of the magnetic and nonmagnetic components the content of the magnetic component in the magnetic product after $k$ stages of separation will be

$$
\beta_{\mathrm{m}}(k)=1-\lambda_{\mathrm{n}}=\frac{\vartheta_{\mathrm{n}}-\alpha_{\mathrm{n}}+\alpha_{\mathrm{n}}\left(1-\vartheta_{\mathrm{n}}\right) \exp \left(-\mu s_{1} k\right)}{\vartheta_{\mathrm{n}}-\alpha_{\mathrm{n}}+\alpha_{\mathrm{n}} \exp \left(-\mu s_{1} k\right)} .
$$

In numerous processes of magnetic enrichment, in the consecutive stages, a pure non-magnetic component is separated. In such a situation the content of the non-magnetic component in the non-magnetic product is equal to $\vartheta_{\mathrm{n}}=1$ and formula (17) can be simplified to the form

$$
\beta_{\mathrm{m}}(k)=\left(1+\frac{\alpha_{\mathrm{n}}}{1-\alpha_{\mathrm{n}}} \exp \left(-\mu s_{1} k\right)\right)^{-1} .
$$

It results from formula (18) that if the relationship of the content of the non-magnetic component $\alpha_{\mathrm{n}}$ to the content of the magnetic component 
$\left(1-\alpha_{n}\right)$ in the feed increases, then the content of the magnetic component in the magnetic product decreases.

Analogously, for $\vartheta_{n}=1$, dependence (16) assumes a simpler form:

$$
\lambda_{\mathrm{n}}\left(k \mid \chi_{\mathrm{n}}\right)=\left(1+\frac{1-\alpha_{\mathrm{n}}}{\alpha_{\mathrm{n}}} \exp \left(\mu s_{1} k\right)\right)^{-1} .
$$

\section{THE EFFECT OF PARTICLE INTERACTIONS AND SEPARATION CONDITIONS ON ENRICIIMENT INDICES}

In all formulas shown above the constant $\mu$ is associated with the physical properties of the mixture components and the separation conditions as well as with the value of particle interactions.

Assuming $\mu=1 / s_{0}\left(s_{0}\right.$ - specific length of the separation path), the quotient $s_{0} / s_{1}$ will be such a number of separations after which the residue of the non-magnetic component in the magnetic product will be 0.33 or the recovery of the non-magnetic component in the non-magnetic product will be 0.67 .

Magnetic separation aims at arranging a set of particles into two or more subsets according to their magnetic properties. In the case of a two-component mixture it is arranged into two subsets. This arrangement requires the work against the forces of particle interactions to be performed. This work is performed by mechanical forces.

Paper [4] contains a derivation of the formula for the number of non-magnetic forces contained in a volume unit of the magnetic stream in a plate separator, after travelling the separation path $x$ :

$$
n(x)=N_{0} \exp \left(-\frac{m g}{V_{\mathrm{c}}} x\right)
$$

where $N_{0}$ is the number of non-magnetic particles in the feed, $V_{\mathrm{c}}$ is the interaction potential of a non-magnetic particle and its surrounding particles, $m$ is the mass of the particle and $g$ the acceleration of gravity.

The exponent numerator of the power of expression (20) contains the force executing the work against particle interacting forces, i.e. the force contributing to the arrangement of particles (in the plate separator it is the force of gravity), while the denominator contains the potential of interactions hindering this arrangement. 
Formula (9) has the same form as formula (20). Consequently, the expression of constant $\mu$ in the above formulae can be expressed as follows:

$$
\mu=\frac{\sum_{i} F_{\mathrm{c}}^{i}}{V_{\mathrm{c}}}
$$

where $\sum_{i} F_{c}^{i}$ represents the sum of all competing forces aiding the exit of non-magnetic particles from the magnetic stream and performing work against the interaction forces. The speed of the arrangement process will therefore depend on the relationship of the values of forces favouring the arrangement to the interactions hindering the arrangement. In order to improve the separation efficiency we should improve this relationship. It can be done either by means of decreasing the particle interactions or by increasing the forces which support the exit of non-magnetic particles from the magnetic stream.

In the case of dry separation the possibilities in this respect are slight. Only the effect of the force of gravity can be improved by an appropriate construction of separators. Applying separators with alternating magnetic field and selecting a proper frequency of the field, the exit of nonmagnetic particles from the magnetic stream is facilitated, without falling of the magnetic particles into the non-magnetic product. In the constant magnetic field, due to the fact that the Van der Waals interaction in the air is much larger than in water, dry magnetic separation is performed for loose materials. Much larger possibilities of control of separation conditions occur in the case of wet separation.

The content of the magnetic component in the magnetic product after a single separation can be calculated from formula (18):

$$
\beta_{\mathrm{m}}(1)=\left(1+\frac{\alpha_{\mathrm{n}}}{1-\alpha_{\mathrm{n}}} \exp \left(-s_{1} \frac{\sum F_{\mathrm{c}}^{i}}{V_{\mathrm{c}}}\right)\right)^{-1}
$$

Applying appropriately high flow rate of the material stream through the separator working space, the mechanical force is enlarged by a hydrodynamic component, increasing consequently the value of constant $\mu$.

Formulas (18) and (22) constitute the basis for analysing the effect of the multiplication factor of separation and particle interactions and 
separation conditions on the quality of the magnetic concentrate, obtained both in low-gradient and high-gradient separators.

\section{VERIFICATION OF THE MODEL}

The model was verified by means of the author's own investigation and literature studies. The author's investigation verified the dependence of the content of the magnetic component in the magnetic product on the number of separations.

It transpires from formula (18) that with the increase of the cleaning separations (stages) the content of the magnetic component in the magnetic concentrate increases. This dependence was verified using natural samples of ilmenite: the ore of particle sizes $0.2-0 ; 0.1-0 ; 0.075-$ 0 and $0.063-0 \mathrm{~mm}$. The magnetite fractions were separated from the samples of the magnetite-ilmenite ore of the above particle sizes by means of the plate separator. The non-magnetic product of this separation, containing mainly ilmenite and the waste rock, constituted the material for further investigation. Samples of the ilmenite ore were subjected to a multi-stage separation in the polygradient separator. The magnetic product of the preceding stage was the feed for the next stage.

The separation conditions in each stage were identical: diameter of steel balls $-10 \mathrm{~mm}$, intensity of external magnetic field $-750 \mathrm{kA} / \mathrm{m}$, height of working space $-0.1 \mathrm{~m}$, velocity of the suspension flow $0.08 \mathrm{~m} / \mathrm{s}$, volume content of solid parts in the suspension $-10 \%$. After each stage of separation in the magnetic product the $\mathrm{TiO}_{2}$ content was determined.

The verification of formula (22) was performed according to the literature data. Therefore the character, resulting from formula (22), of the dependence of the content of magnetic component in the magnetic product (after a single separation) on the value of competing forces and particle interactions was verified.

The value of the sum of mechanical forces in the high-gradient separator is affected by the flow velocity of suspension through the separator working space and the intensity of vibrations of fibres of the separator matrix (explained further in the text). Therefore the dependence of the content of magnetic component on flow velocity and on the intensity of the current inducing the vibrations of fibres was investigated. 
Particle interactions can be divided into magnetic and surface interactions. Magnetic interactions in the separator working space (at the constant ore composition and fixed conditions of separation) depend on the content of solid parts in the suspension directed to the separator. Therefore the dependence of the content of the magnetic component in the magnetic product of the drum separator on the content of solid parts in the suspension was given.

\section{ANALYSIS OF RESULTS}

The influence of the number of stages on the results of separation

Figures 1-4 show the dependence of $\mathrm{TiO}_{2}$ content in the magnetic concentrate on the effective separation path measured in $s_{1}$ units. Due to the fact that the verification of the dependence of the content of the magnetic component in the magnetic product on the multiplication factor of separation was performed on the ilmenite ore samples in which the degree of ilmenite liberation, depending on particle size, is smaller than 1, dependence (18) should be written in the following form:

$$
\beta_{\mathrm{m}}(k)=\left(1+\frac{\alpha_{\mathrm{n}}}{1-\alpha_{\mathrm{n}}} \exp \left(-\mu s_{1} k\right)\right)^{-1} \beta_{\mathrm{t}}
$$

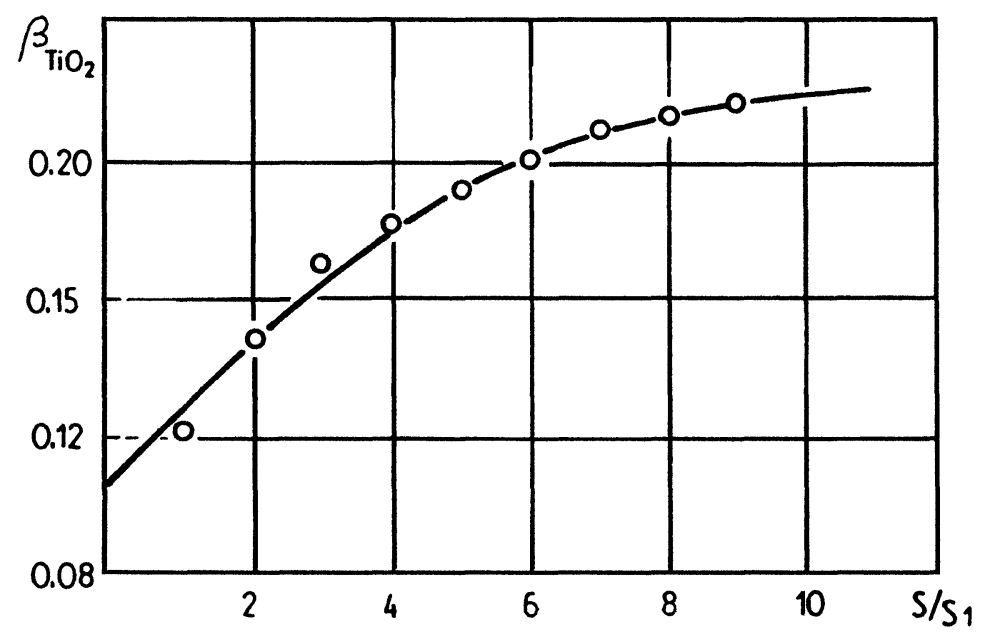

FIGURE 1 Dependence of the $\mathrm{TiO}_{2}$ content in the magnetic product on the number of separations. Size fractions $0.2-0 \mathrm{~mm}$. 


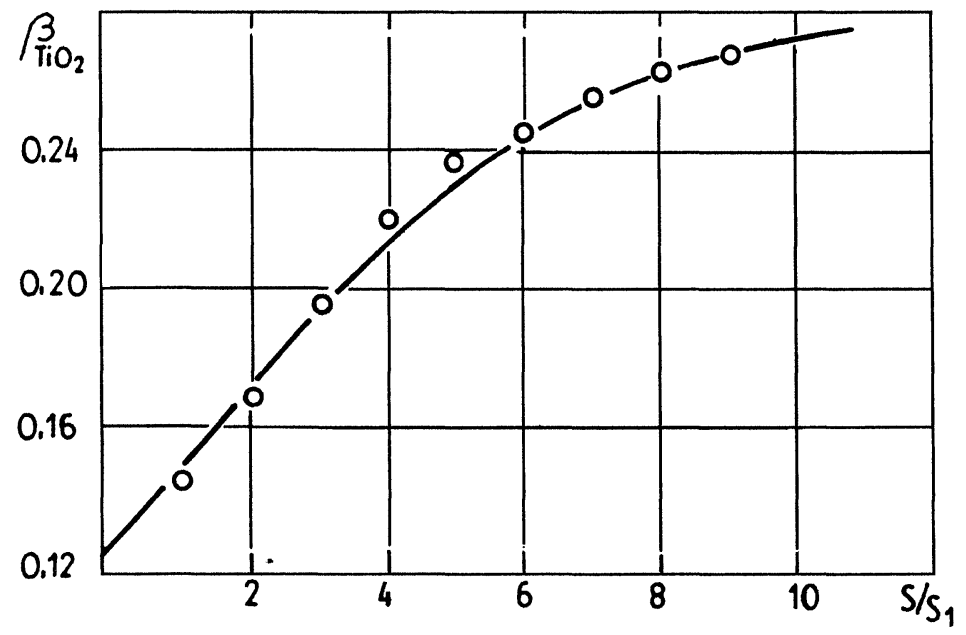

FIGURE 2 Dependence of the $\mathrm{TiO}_{2}$ content in the magnetic product on the number of separations. Size fractions $0.1-0 \mathrm{~mm}$.

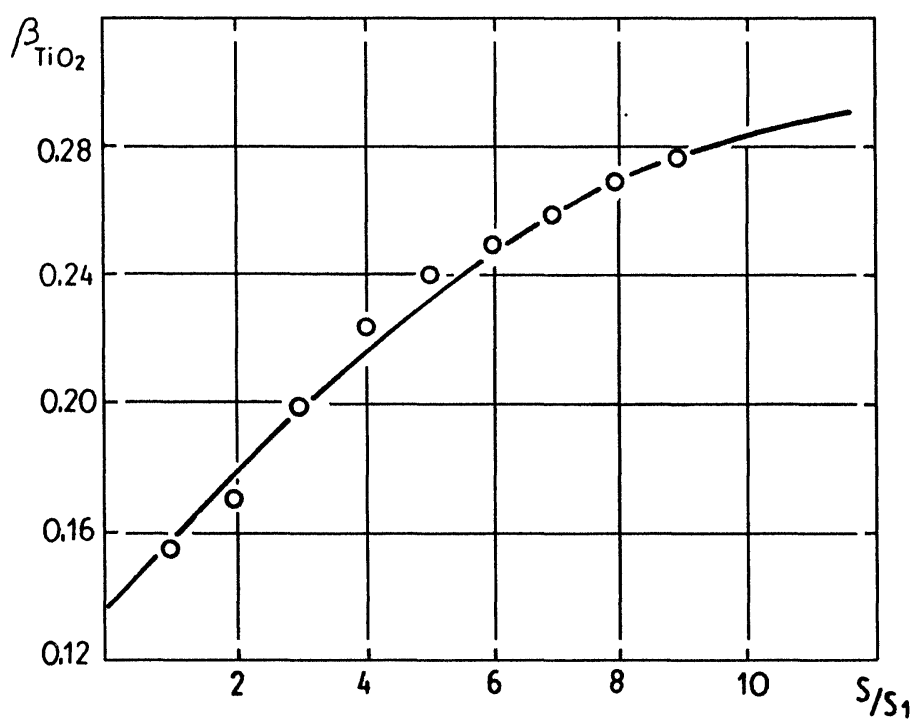

FIGURE 3 Dependence of the $\mathrm{TiO}_{2}$ content in the magnetic product on the number of separations. Size fractions $0.075-0 \mathrm{~mm}$. 


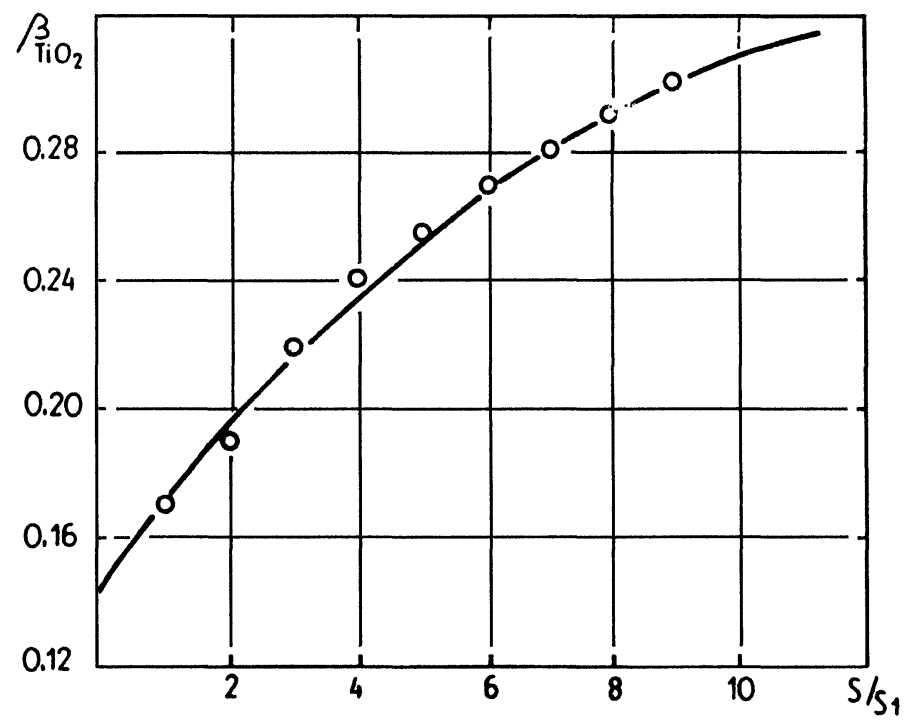

FIGURE 4 Dependence of the $\mathrm{TiO}_{2}$ content in the magnetic product on the number of separations. Size fractions $0.06-0 \mathrm{~mm}$.

TABLE I The values of parameters of Eq. (23)

\begin{tabular}{lcccc}
\hline Class $(\mathrm{mm})$ & $\mu(1 / \mathrm{m})$ & $\beta_{\mathrm{t}}$ & $\alpha_{\mathrm{n}}$ & $R$ \\
\hline $0.2-0$ & 3.61 & 0.226 & 0.520 & 0.9941 \\
$0.1-0$ & 3.44 & 0.284 & 0.559 & 0.9935 \\
$0.075-0$ & 2.71 & 0.308 & 0.554 & 0.9900 \\
$0.06-0$ & 2.53 & 0.340 & 0.552 & 0.9915 \\
\hline
\end{tabular}

where $\beta_{\mathrm{t}}$ represents the theoretically possible maximum, at a given size reduction, of the $\mathrm{TiO}_{2}$ content in the magnetic concentrate. Values of $\beta_{\mathrm{t}}$, $\mu$ and $\alpha_{\mathrm{n}}$ were determined by fitting the empirical data to the model dependence (23).

Table I presents the values of the calculated parameters. The solid lines in Figs. 1-4 were drawn according to the model dependences of Table I.

High values of the correlation coefficient $R$ indicate a very good compatibility of the model with the experimental data. A significant decrease of the rate separation constant $\mu$ for the particles below $0.075 \mathrm{~mm}$ proves an appearing influence of particle interactions. With 
the increase of size reduction the number of separations necessary for exceeding a certain threshold of content, referring to the content $\beta_{\mathrm{t}}$, increases. At a higher size reduction, as it can be seen in Table I, the content of $\mathrm{TiO}_{2}$ increases in the purest particles of the magnetic product ( $\beta_{\mathrm{t}}$ grows) which is associated with the growth of magnetic susceptibility of magnetic particles. With the increase of susceptibility of magnetic particles, the length of the separation path and consequently, the number of separations increases [4]. In order to reach the content $\beta=0.95 \beta_{\mathrm{t}}$ it is necessary, in case of the particles $-0.2-0 \mathrm{~mm}$, to carry out nine separations while in case of the particles smaller than $0.06 \mathrm{~mm}$ fourteen separations are required.

\section{The effect of the flow velocity suspension on the separation results}

To analyse the dependence of the separation results on the flow velocity of suspension through the matrix of the polygradient separator, the rate separation constant $\mu$, according to formula (21), can be expressed in the following way:

$$
\mu=s_{1} \frac{m g+6 \pi \eta a v}{V_{\mathrm{c}}}=B+C v
$$

where $\eta$ is the coefficient of dynamic viscosity of a liquid; $B$ and $C$ the constants given by

$$
B=\frac{s_{1} m g}{V_{\mathrm{c}}}, \quad C=\frac{6 s_{1} \pi \eta a}{V_{\mathrm{c}}} .
$$

It was simultaneously assumed that the movement of a liquid with velocity $v$ is of a laminar character and the force of hydrodynamic resistance is expressed by Stokes' formula. Therefore the sum of the competing forces contains two components; i.e. the force of gravity and the force of hydrodynamic resistance.

The dependence of the content of the useful component in the magnetic concentrate on the flow velocity of suspension will thus be as follows:

$$
\beta_{\mathrm{m}}(v)=(1+b \exp (-c v))^{-1} \beta_{\mathrm{t}}
$$


where $b=\left[\alpha_{\mathrm{n}} /\left(1-\alpha_{\mathrm{n}}\right)\right] \exp (-B)$ while $\beta_{\mathrm{t}}$ represents the equilibrium content of the component of the particles of the magnetic concentrate of the magnetic susceptibility determined from the condition of equilibrium of the competing and magnetic forces in the working space of the separator. Figure 5 shows the dependence of the Fe content in the magnetic concentrate of the oxidized iron ore (taconite) on the flow velocity of suspension through the working space of the separator $[5,6]$. An investigation was carried out in the high-gradient separator at the magnetic field intensity $1600 \mathrm{kA} / \mathrm{m}$. The working space of the separator (matrix) was filled with steel wool of the packing density of $5 \%$. The content of $\mathrm{Fe}$ in the feed was $\alpha_{\mathrm{Fe}}=35 \%$ while the particle size distribution of the ore was below $20 \mu \mathrm{m}$. The maximum content of iron in the magnetic concentrate which was reached at the enrichment of this ore was $65 \%$ [5]. It can be therefore assumed that $\beta_{\mathrm{t}}=65 \%$. Taking into consideration the fact that $\beta_{\mathrm{m}}(0)=\alpha=35 \%$, the following dependence was obtained on the basis of the data of Fig. 5 (continuous line on the graph):

$$
\beta_{\mathrm{m}}(v)=65(1+0.857 \exp (-0.065 v))^{-1}
$$

in which $v$ is expressed in $\mathrm{cm} / \mathrm{s}$ while $\beta_{\mathrm{m}}$ in $\%$.

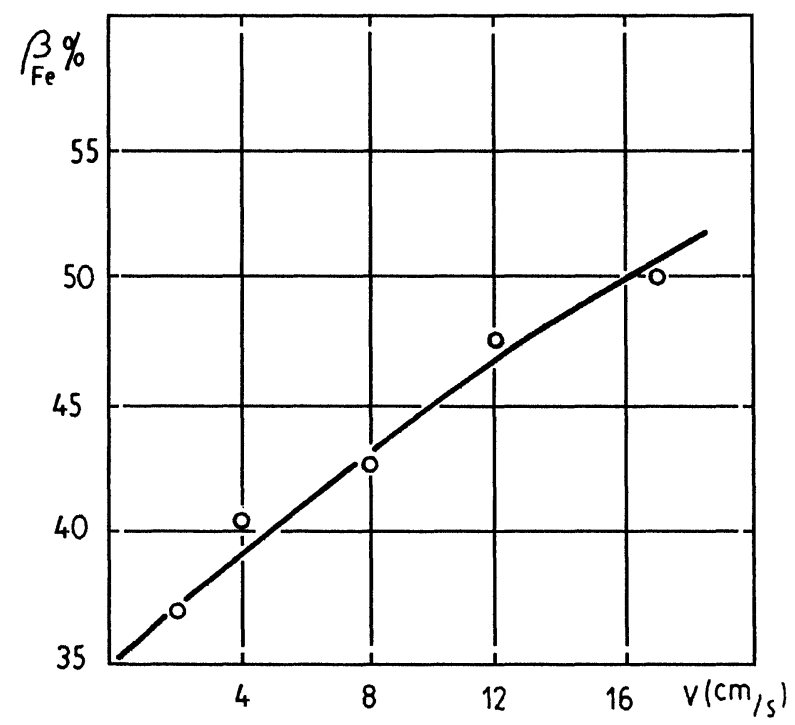

FIGURE 5 Dependence of the $\mathrm{Fe}$ content in the concentrate on the flow velocity of suspension. 
As can be seen from Fig. 5 the compatibility of the experimental data with the model dependence is satisfactory.

\section{The influence of the matrix vibrations on the separation results}

In order to intensify the process removal of non-magnetic particles from the working space of high-gradient separators filled with steel wool, the steel wool fibres are made to vibrate with appropriate frequency, perpendicular to the direction of the external magnetic field [7]. Vibrations of frequency contained in the range of sound frequencies are induced by an alternating magnetic field induced in the coil wound on the steel wool vessel (Fig. 6). These vibrations on the one hand keep the magnetic floc loosened while, on the other, as a result of inertia, they pass the momentum of a vibrating fibre to non-magnetic particles, facilitating them to leave the floc structure.

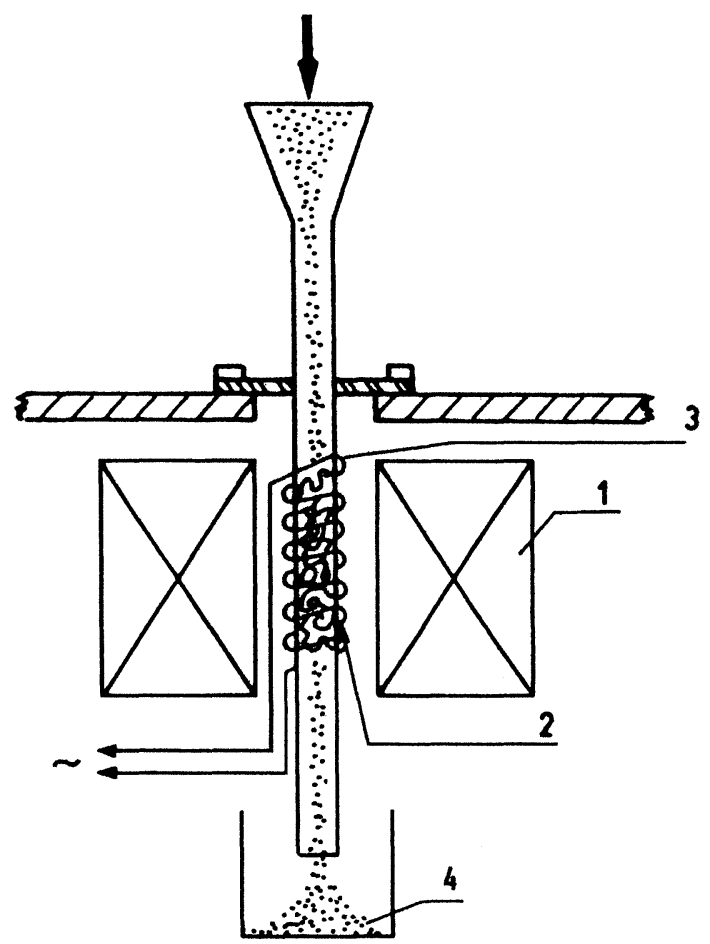

FIGURE 6 The polygradient separator with a vibrating matrix: 1 - magnet, 2 matrix, 3 - coil inducing fibre vibrations, 4 - tank of the non-magnetic product. 
The force of the fibre vibrations is proportional to the force with which the magnetic field of the coil acts on the fibre, i.e. $F \sim H^{2}$ [8]. Since the intensity of the magnetic field of the coil is proportional to the intensity of the current in the coil winding, then $F \sim I^{2}$. A floc is connected with a vibrating fibre and therefore the volume will be affected by the force of inertia, proportional to $I^{2}$ and parallel to the flow direction. In this situation the sum of competing forces occurring in expression (21) will be increased by another component, proportional to $I^{2}$. Assuming that the remaining elements in the expression (21) are constant, the dependence of the content of the magnetic component in the magnetic concentrate will be expressed due to Eq. (22) by a formula similar to formula (25):

$$
\beta_{\mathrm{m}}(I)=\left(1+b \exp \left(-c I^{2}\right)\right)^{-1} \beta_{\mathrm{t}} .
$$

The investigation of the effect of the vibrations of the matrix fibres on the results of magnetic separation were performed on mixtures of wolframite and quartz of the concentration $0.5 \% \mathrm{WO}_{3}$, particle size distribution of wolframite $0-5 \mu \mathrm{m}$ and quartz $0-76 \mu \mathrm{m}$ [7]. The intensity of the external (constant) magnetic field was $1200 \mathrm{kA} / \mathrm{m}$ while the flow velocity of suspension through the working space of the separator was $1.4 \mathrm{~cm} / \mathrm{s}$. The matrix consisted of steel wool of rectangular cross section of fibres of $150 \times 50 \mu \mathrm{m}$.

Figure 7 presents the dependence of the wolframite content in the magnetic concentrate on the intensity of the vibration current. This dependence is approximated by the formula

$$
\beta_{\mathrm{m}}(I)=35.54\left(1+3.49 \exp \left(-0.45 I^{2}\right)\right)^{-1} .
$$

The continuous line of Fig. 7 was drawn according to expression (28). Therefore the compatibility of the model with the experiments is very good. By means of a proper choice of the vibration intensity and the flow velocity of suspension it is possible to reach a very high selectivity of separation, confirmed in industrial conditions by the separation of cassiterite ore [7].

\section{The effect of the concentration of solids in the suspension}

The volume concentration of solids in the suspension fed into the separator exerts a direct influence on the value of magnetic interactions 


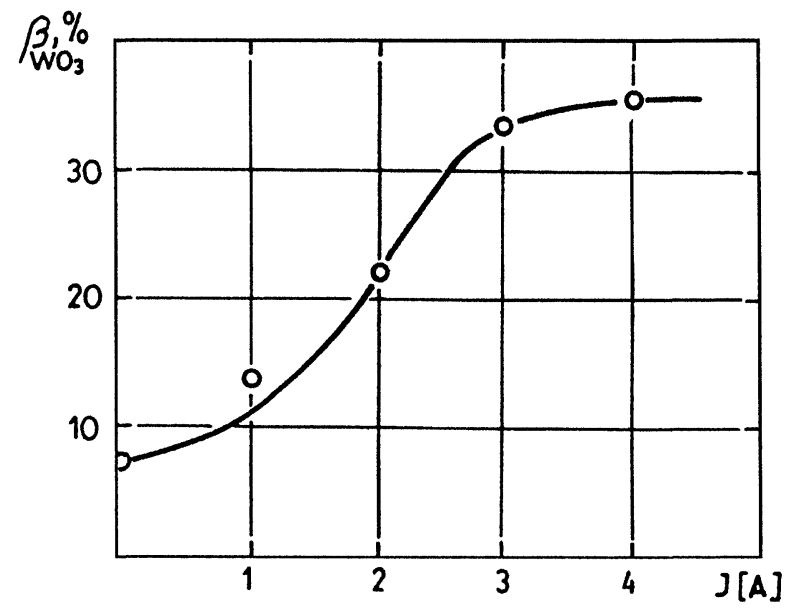

FIGURE 7 Dependence of the wolframite content in the concentrate on the intensity of the vibration current.

between the magnetic particles. Starting from Coulomb's magnetostatic law Berdichevskiy and Karmazin presented a formula for potential of magnetic interactions $V_{\mathrm{m}}$ of two particles of the same size and the same magnetic susceptibility [9]:

$$
V_{\mathrm{m}}=-\frac{\chi_{\mathrm{m}}^{2} H^{2} S^{2}}{k_{1} r\left(1+\chi_{\mathrm{m}} N\right)}
$$

where $\chi_{\mathrm{m}}$ is the volume magnetic susceptibility, $H$ the intensity of the external magnetic field, $S$ the area of the transverse cross-section of a particle, $N$ the demagnetization factor, $r$ the distance between particles, and $k_{1}$ a constant depending on the assumed system of units.

It transpired from formula (29), that the potential of magnetic interactions between two magnetic particles in the Coulomb-type approximation is proportional to $r^{-1}$ ( $r$-distance between particles). Next, the average distance between magnetic particles is proportional to the reciprocal value of the third root from the volume concentration of magnetic particles in suspension [10]. Thus, formula (29) can be written as follows:

$$
V_{\mathrm{m}} \sim-\frac{\chi_{\mathrm{m}}^{2} S^{2} H^{2} c_{\mathrm{m}}^{1 / 3}}{\left(1+\chi_{\mathrm{m}} N\right)^{2}}
$$


where $c_{\mathrm{m}}$ is the volume concentration of the magnetic component in the suspension. If the volume content of the magnetic component in the dry feed is equal to $\alpha_{m}=1-\alpha_{n}$ and the volume concentration of solids in the suspension is $Z$, then the volume concentration of the magnetic component in the suspension will be $c_{\mathrm{m}}=\alpha_{\mathrm{m}} Z$. Assuming that both the composition of the feed for the separation process, i.e. the relation $\alpha_{\mathrm{n}} /$ $\left(1-\alpha_{n}\right)$, and also the values of all competing forces are constant and neglecting all other particle interactions (except the magnetic one, Eq. (30)) expression (18), after considering Eqs. (21) and (30), can be written as follows:

$$
\beta_{\mathrm{m}}(k \mid Z)=\left(1+\frac{\alpha_{\mathrm{n}}}{1-\alpha_{\mathrm{n}}} \exp \left(-\frac{B k}{Z^{1 / 3}}\right)\right)^{-1} \beta_{\mathrm{t}}
$$

where $B$ is a constant given by

$$
B=\frac{s_{1}\left(1+\chi_{\mathrm{m}} N\right)^{2} \sum F_{\mathrm{c}}^{i}}{\chi_{\mathrm{m}}^{2} S^{2} H^{2}\left(1-\alpha_{\mathrm{n}}\right)^{1 / 3}} .
$$

It results from formula (31) that with the increase of the concentration of solids in the suspension and in order to obtain a concentrate of the required quality, more cleaning separation stages should be performed than in the case of lower concentration of solids. It is confirmed in practical enrichment operations [11,12].

In a single separation the increase of the concentration of solids decreases the quality of the magnetic concentrate according to the equation

$$
\beta_{\mathrm{m}}(Z)=\left(1+\frac{\alpha_{\mathrm{n}}}{1-\alpha_{\mathrm{n}}} \exp \left(-\frac{B}{Z^{1 / 3}}\right)\right)^{-1} \beta_{\mathrm{t}} .
$$

The investigation of the effect of the content of solids in the suspension were carried out using the iron ore of Krivoy Róg [11] of particle size distribution $0.1-1 \mathrm{~mm}$ and $\mathrm{Fe}$ content $\alpha_{\mathrm{Fe}}=22 \%$. The pure magnetic concentrate contained about $65 \% \mathrm{Fe}$. This value can be assumed as equal to $\beta_{\mathrm{t}}$.

The magnetic separation tests were carried out using a drum seperator of drum dimensions $400 \times 400 \mathrm{~mm}$ with a three-pole magnetic system. The magnetic field intensity on the surface was $112 \mathrm{kA} / \mathrm{m}$ and 
the drum rotational speed was $54 \mathrm{rpm}$. In industrial operations the concentration of solids is given as percentage by weight. The weight concentration $Z_{\mathrm{m}}$ is recalculated into the volume concentration according to the following formula:

$$
Z=\frac{b}{\left(a-Z_{\mathrm{m}}\right) \rho_{k}} Z_{\mathrm{m}}
$$

where

$$
a=\frac{\rho_{k}}{\rho_{k}-\rho_{0}}, \quad b=\frac{\rho_{0} \rho_{k}}{\rho_{k}-\rho_{0}},
$$

$\rho_{\mathrm{k}}$ being the average density of the dry feed, and $\rho_{0}$ the density of water.

Figure 8 presents the dependence of the $\mathrm{Fe}$ content in the magnetic concentrate as a function of the volume concentration of solids in the suspension fed into the separator. The experimental points have been taken from work [11]. The solid line represents the following dependence:

$$
\beta_{\mathrm{Fe}}(Z)=65\left(1+1.94 \exp \left(-1.2 Z^{-1 / 3}\right)\right)^{-1} .
$$

The dependence $\beta_{\mathrm{Fe}}(Z)$ was calculated for the Coulomb-type interaction between the magnetic particles. The experimental data are in good agreement with the model for the Coulomb-type approximation of interactions while they differ completely from the dependence drawn for the dipole-dipole interaction.

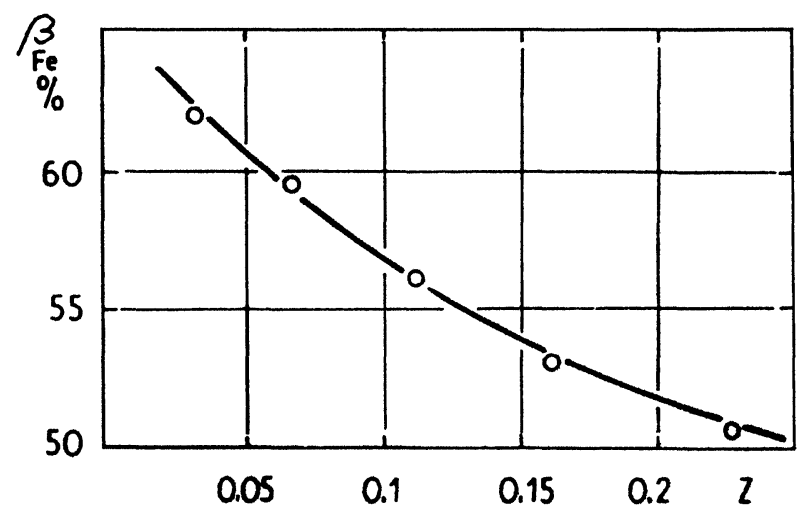

FIGURE 8 Dependence of the $\mathrm{Fe}$ content in the concentrate on the volume concentration of solids in the suspension. 


\section{Acknowledgement}

The author is grateful to the Scientific Research Committee for the funding of this research through Grant No. 9 T12A 01514.

\section{References}

[1] Siwiec, A. Górnictwo 6, 195 (1982) (in Polish).

[2] Rozanov, J.A. Introduction to the Theory of Stochastic Processes (in Polish). Polish Scientific Publisher, Warsaw, 1974.

[3] Stępiński, W. Gravity Concentration (in Polish). Polish Scientific Publisher, Warsaw, 1964.

[4] Brożek, M. Magnetic and Electrical Separation 9 (1998).

[5] Hays, R.M. SME Transaction 244, 331 (1969).

[6] Oberteuffer, J.A. IEEE Trans. Magn 9, 303 (1973).

[7] Zhongyuan, Sun and Zhengnan, Li: Proc. XV IMPC. Cannes, 1985, p. 410.

[8] Svoboda, J. Magnetic Methods for the Treatment of Minerals. Elsevier Sci. Publ., Amsterdam-Oxford-New York-Tokyo, 1987.

[9] Berdichevskiy, R.J. and Karmazin, V.V. Obogascenie rud (in Russian). no. 2, 41 (1968).

[10] Barfod, N. Pow. Techn. 6, 39 (1972).

[11] Egorov, N.F. Obogascenie rud (in Russian). no. 2, 5 (1964).

[12] Egorov, N.F. Obogascenie rud (in Russian). no. 3, 32 (1969). 